\title{
Por una epistemología de la Salud Colectiva: los conceptos sociológicos
}

\author{
For an epistemology of Collective Health: \\ the sociological concepts
}

Everardo Duarte Nunes ${ }^{1}$

\footnotetext{
${ }^{1}$ Licenciado en Ciencias Sociales. Doctor en Ciencias, Universidade Estadual de Campinas (Unicamp). Profesor Colaborador de la Facultad de Ciencias Médicas, Unicamp, Brasil.

evernunes@uol.com.br
}

RESUMEN A partir de conceptos básicos de la sociología, se traza una elaboración teórica que busca una primera aproximación para la construcción de una epistemología de la salud colectiva. El texto expone, inicialmente, una conceptualización de la epistemología, recordando que las diversas epistemologías -fenomenológica, constructivista, histórica y arqueológica- han iluminado la construcción científica de la Salud Colectiva. Si bien los desdoblamientos de estas disciplinas no son abordados, el análisis enfatiza como punto de partida las nociones de concepto, constructos y categorías analíticas. Se destacan tres conceptos fundantes de las ciencias sociales, que se convierten en categorías analíticas: colectivo, sociedad y estructura. Estos conceptos son articulados de forma interactiva con otros tres: individuo, representación y acción. Se apuntan las principales características de esos conceptos y la interrelación entre ellos, así como la idea de que se pueden desprender otros conceptos de esta base conceptual. Se enfatiza la cuestión de superar las oposiciones (objetivo/subjetivo, colectivo/individual) y la necesidad de pensar conjuntamente aspectos de la realidad, clásicamente entendidos como antagónicos.

PALABRAS CLAVE Epistemología; Sociología; Sociología Médica, Salud Pública.

ABSTRACT From basic concepts of sociology, a theoretical elaboration is outlined which looks for a first approach for the building of an epistemology of Collective Health. The paper exposes initially a conceptualization of epistemology, taking into account that different epistemologies - phenomenological, constructivist, historical and archeologicalhave illuminated the scientific construction of Collective Health. Although the split of these disciplines are not taken into account, the analysis emphasizes the notions of concept, constructs and analytical categories as a starting point. Three founding concepts in the social sciences are stressed, which turn into analytical categories: collective, society and structure. These concepts are articulated in an interactive way with three others: individual, representation and action. The main characteristics of these concepts and their interrelation are highlighted together with the idea that other concepts may derive from this conceptual basis. The question of overcoming the oppositions is emphasized (objective/subjective, collective/individual) along with the need to think together these aspects of reality, which were classically understood as antagonists.

KEY WORDS Epistemology; Sociology; Sociology, Medical; Public Health. 


\section{INTRODUCCIÓN}

La Salud Colectiva heredó categorías y conceptos de diversos campos de saberes; especialmente de la biología, la sociología, la antropología, las ciencias políticas, la ciencia de la administración, la historia, el psicoanálisis, la epidemiología, por nombrar solo algunas de las disciplinas que contribuyeron a la construcción conceptual de ese campo. En este trabajo (a) abordamos, en forma particular, la vertiente sociológica y su importancia en el dimensionamiento de las categorías analíticas que posibilitan una aproximación a los objetos tratados en el campo de la Salud Colectiva.

Pensamos que, para una mejor comprensión de esta compleja cuestión, es necesario definir inicialmente, aunque sea de forma abreviada, qué se entiende por epistemología. Según Japiassu (1), en un sentido bien amplio del término, podemos considerarla como "el estudio metódico y reflexivo del saber, de su organización, formación, desarrollo, funcionamiento y de sus productos intelectuales"; y clasifica la epistemología en:

...global (general), cuando trata del saber globalmente considerado, con la virtualidad y los problemas del conjunto de su organización, sean "especulativos", o "científicos"; particular, cuando trata de un campo particular del saber, sea "especulativo", o "científico"; específica, cuando trata de una disciplina intelectualmente constituida en una unidad bien definida de saber, y de estudiarla de modo próximo, detallado y técnico, mostrando su organización, su funcionamiento y las posibles relaciones que ella mantiene con las demás disciplinas. (1 p.16)

La epistemología puede ser interna de una ciencia, cuando se analizan críticamente los procedimientos del conocimiento que ella utiliza, con el objetivo de establecer los fundamentos de la disciplina; y derivada, cuando se realiza un análisis de la naturaleza de los procedimientos del conocimiento de una ciencia para saber cómo esta forma de conocimiento es posible, así como para determinar la parte que le cabe al sujeto y al objeto en el modo particular de conocimiento que caracteriza a la ciencia. Según Japiassu, en el interior de esas dos categorías -sujeto y objeto- hay subclases:

...conforme el acuerdo suponga un primado del

Objeto, que se impone al espíritu (conocimiento sacado del objeto), un primado del Sujeto (conocimiento sacado del sujeto) que antecede al objeto, o una interacción entre el Sujeto y el Objeto. [...] las epistemologías actualmente vivas y significativas están centradas sobre las interacciones del Sujeto y del Objeto: la epistemología fenomenológica, ilustrada por Husserl; la epistemología constructivista y estructuralista, ilustrada por Piaget; la epistemología histórica, ilustrada por Bachelard; la epistemología "arqueológica", ilustrada por Foucault. (1 p.22)

Se puede decir que las epistemologías anteriormente enunciadas no son extrañas al campo de la Salud Colectiva y han iluminado sus caminos en términos de su construcción científica. Incluso hay autores que profundizaron en esas vertientes, buscando develar la construcción del "pensar en salud", en la expresión de Testa (2), de elaborar una "teoría de la salud", como lo hace Samaja (3), o de repensar "críticamente la ciencia", como Breilh (4). Todos coinciden en señalar que el trabajo epistemológico en la salud es extremadamente complejo, pues él es presionado por la reconceptualización del propio objeto científico "salud". Para Breilh, "al Ilegar a su fin el siglo XX e iniciar el nuevo milenio con una crisis del pensamiento de la modernidad, la trama epistémico-contemporánea se muestra plena de confrontaciones" (4 p.55). Cita, entre éstas, a la oposición entre aquellos que defienden la preeminencia de las concepciones totalizantes o grandes narrativas sobre la totalidad, por un lado, y aquellos que se vuelven a favor de perspectivas que enfatizan lo micro, lo local, la diversidad de las narrativas, por otro. Para Breilh, esta es una falsa oposición entre "la dictadura de la totalidad" y la "tiranía del fragmento". En este sentido, el debate sobre temas cruciales, como los de "objeto" y "sujeto" del conocimiento, la relación entre lo macro y lo micro en la determinación de la realidad, la propia relación entre lo determinado y lo incierto o contingente, las visiones opuestas sobre la complejidad tienen repercusiones 
sobre la discusión teórico-metodológica de la epidemiología en el caso analizado por Breilh (4), pero que se aplican, en toda su extensión en la Salud Colectiva.

Si bien no volveremos nuevamente sobre estas cuestiones, vamos a detenernos en algunos conceptos que nos parecen fundamentales en la estructuración del campo científico de la Salud Colectiva. Para esto, estableceremos algunas definiciones previas que orientarán la estructura de esta presentación. Asumimos, como punto de partida, la idea bien definida por Mendonça cuando señala que:

Los conceptos son construcciones lógicas, establecidas de acuerdo con un cuadro de referencias. Adquieren su significado dentro del esquema del pensamiento en el cual son colocados.

[...] Cada ciencia usa sus propios conceptos para la comunicación de sus conocimientos. (5 p.15)

En este sentido, sistema teórico y sistema conceptual son equivalentes. Así, "a través de un dispositivo conceptual, se busca representar el fenómeno o aspectos del fenómeno que ocurren en el mundo real, una representación resumida de un conjunto de hechos". Estamos entendiendo el concepto como "una palabra que expresa una abstracción formada por la generalización a partir de específicos" (5 p.15-16).

Mendonça, dice que la función de los conceptos es representar la realidad o aspectos de la realidad, y sigue afirmando:

\footnotetext{
Algunos conceptos están muy próximos de los fenómenos que representan; otros no pueden unirse directamente a los hechos $\mathrm{u}$ objetos que quieren representar. Son inferencias de la realidad, pero, en nivel más elevado de abstracción, son también conceptos, pero con un significado adicional, de haber sido deliberado y conscientemente inventado o adoptado para un objetivo científico específico de análisis. (5 p.16)
}

Sin duda, las ideas expuestas nos conducen a aquello que los filósofos consideran los dos problemas fundamentales de la noción de concepto: su naturaleza y su función. Según Abbagnano (6 p.151), dos fueron las soluciones fundamentales sobre el problema de la naturaleza: según la primera, el concepto es la esencia de las cosas y, precisamente, su esencia necesaria, por la cual ellos no pueden existir de modo diferente de aquello que son; según la segunda, el concepto es un signo. La primera acepción viene del período clásico de la filosofía griega, que retorna en la fenomenología de Husserl. Como signo, tiene también sus vertientes griegas y escolásticas, pero como destaca Abbagnano (6 p.155), "el término concepto, o significado, aparece más frecuentemente referido para indicar la connotación que la denotación". Con relación a la función del concepto, destacamos que puede ser concebida como final y como instrumental. Reproduciendo a Abbagnano:

La función final atribuye al concepto, su interpretación como esencia: ya que por esta interpretación el concepto no tiene otra función que la de exprimir o revelar la sustancia de las cosas. La función se identifica, desde este punto de vista, con la propia naturaleza del concepto. (6 p.155)

Se destacan como funciones: describir los objetos de la experiencia para permitir su reconocimiento; clasificar los hechos en los conceptos; organizar los datos de la experiencia, con el objetivo de establecer entre ellos conexiones de naturaleza lógica; prever el concepto como medio de anticipación o proyección. De estas dos últimas funciones nacen en el campo científico los modelos y las construcciones.

Estas observaciones son importantes, porque en el caso de la Salud Colectiva se observa que estas dos situaciones pueden estar presentes. Si bien no desarrollaremos estas cuestiones, adoptaremos como punto de partida para la organización de los conceptos la idea de las "abstracciones de nivel más elevado". Para Mendonça (5), esas "abstracciones" son denominadas "construcciones conceptuales, o 'constructos', porque son construidas a partir de conceptos de nivel inferior de abstracción". En realidad, la pretensión es la de "delinear algunas claves interpretativas", o "categorías analíticas", siguiendo, de alguna forma, la propuesta de Domingues (7) que nos aporta enriquecedoras ideas para entender la construcción de la teoría sociológica. Para este autor: 
...las categorías analíticas no existen en la realidad, la cual consiste en el entrelazamiento de numerosos fenómenos concretos; las categorías analíticas son conceptos forjados por los sociólogos con el objetivo de, por medio de la abstracción del entrelazamiento de los fenómenos concretos, poder observar aspectos discretos y específicos de la realidad social. (7 p.17)

\section{CONCEPTOS SOCIOLÓGICOS FUNDANTES}

Las nociones anteriores nos parecen adecuadas, pues trabajaremos con tres conceptos fundantes de las ciencias sociales, que se convierten en categorías analíticas: colectivo, sociedad, estructura, que, a su vez, pueden ser articuladas de forma interactiva con otros tres conceptos: individuo, representación, acción.

Sin duda, el concepto de colectivo es central para el campo de la Salud Colectiva, como lo fue para la sociología que desde sus primeros momentos, como bien analiza Corcuff (8), fue marcada por la oposición entre lo colectivo y lo individual, "la sociedad" y "el individuo".

Al proponer la sociología como un campo específico de conocimiento, Durkheim (9) valorará lo colectivo (o lo social), como algo distinto de lo individual. Para él,

...la sociedad no es una simple suma de individuos, pues el sistema formado por la asociación de estos representa una realidad específica que tiene sus propias características. Sin duda que nada colectivo se puede producir sin que se manifiesten las conciencias particulares; pero esta condición necesaria no es suficiente. Es necesario aun que estas conciencias se asocien, de una cierta manera; es de esta combinación que resulta la vida social y, por consiguiente, es esta combinación la que la explica. (9 p.139)

Las discusiones que se seguirán a lo largo de los años replantearán el problema de las partes y del todo, del individuo y del colectivo, y como escribe Corcuff (8), o: "contra todo al mismo tiempo, contra el holismo y el individualismo, las nuevas sociologías tienden entonces a aprehender individuos plurales producidos y productores de relaciones sociales variadas". Esta es una cuestión central de la sociología y será retomada por la Salud Colectiva desde las primeras discusiones sobre el campo.

En un texto que se tornaría referencia, Donnangelo decía que:

La imprecisa designación de colectivo ha recubierto efectivamente distintos objetos de saber e intervención. Es verdad que esta noción ha sido frecuentemente utilizada de manera acrítica para designar un campo supuestamente uniforme de ocurrencias, sea porque lo colectivo aparezca como contrapuesto a lo individual (colección), sea porque pretenda ser indicativo de los efectos sociales generados por la presencia de determinados fenómenos. (10 p.20)

Al analizar la producción científica producida, señala que la diversidad temática, el énfasis en distintos objetos y en distintos campos de saber, por variaciones temáticas y metodológicas del análisis, por distintas opciones sobre métodos experimentales, reproducían

\footnotetext{
...la amplitud posible de la gama de connotaciones asumidas por la noción de colectivo: colectivo/medio; colectivo/conjunto de individuos; colectivo/interacción entre elementos de; colectivo como conjunto de efectos o consecuencias de la vida social; colectivo transformado en social como campo específico y estructurado de prácticas. (10 p. 27)
}

En realidad, el soporte de la discusión, como fue propuesta por Pereira (11 p.3), es la de entender que el objeto de tal disciplina (refiriéndose a la Medicina Social) "no sería representado por cuerpos biológicos, sino por cuerpos sociales, grupos y clases sociales y relaciones sociales referidas al proceso salud-enfermedad".

Se percibe que, a lo largo de su historia, la Salud Colectiva viene buscando un mejor entendimiento de su objeto central estableciendo, incluso, más recientemente, un contrapunto con la cuestión del sujeto y de la subjetividad. En ese sentido, acompaña las discusiones contemporáneas desarrolladas por la sociología, que recupera la crítica de la oposición entre individuos y sociedad, 
como propone Norbert Elias (12) -cuando dice que ni el individuo es una entidad exterior a la sociedad, ni la sociedad una entidad exterior a los individuos, y que la sociología tiene como objeto individuos interdependientes-; por Pierre Bourdieu (13) que, al establecer la relación entre "estructura" y "acción", atribuye mayor peso a la primera pero sin descuidar la segunda y define "campo" y "habitus" exteriorizando la interioridad de los procesos; por Anthony Giddens (14) que, al conceptualizar dualidad estructural, señala que "las propiedades estructurales de los sistemas sociales son simultáneamente condiciones y resultados de las actividades realizadas por los agentes que forman parte de estos sistemas". Como analiza Corcuff (8), esos tres autores transitan desde las "estructuras sociales hasta las interacciones" y dan "un cierto predominio a las estructuras sociales y a los aspectos macrosociales de la realidad, integrando de manera variable, las dimensiones subjetivas e interaccionales", de un modo diferente de aquellos que transitarán "desde las interacciones a las estructuras sociales", como es el caso de Peter Berger y Thomas Luckman (15), y Aaron Cicourell (16).

Esta discusión, como ya fue señalado, ha sido asimilada por la Salud Colectiva; así, "el sujeto y la subjetividad en la praxis de la salud" fue ampliamente tratado en un número temático de la revista Ciencia \& Saúde Coletiva (17). La importancia de este debate fue producto del texto de Cecília Minayo, al señalar que "el conocimiento médico y de la salud pública han sido sistemáticamente marcados por el apagamiento del sujeto" (18 p.14) y muchas corrientes, al enfatizar las aproximaciones macroestructurales y la crítica a los aparatos del Estado, dejaron en "segundo plano el análisis de la praxis y de los sujetos sociales, históricos y culturales en la configuración del campo" de la Salud Colectiva (18 p.15). Como recuerda la autora, a partir de los años '90 hay un "retorno del sujeto" y con él la posibilidad de traer a la escena al sujeto "como actor de las reformas" (18 p.15). Es evidente la importancia de este abordaje, y los diversos trabajos elaborados que fueron en parte sintetizados en ese número de la revista comprueban esa preocupación por los "agenciamientos tecnosemiológicos", por el campo de la Salud Mental, por la formación médica, por las organizaciones de salud, por el planeamiento, por la epidemiología.
La noción de colectivo nos conduce a otras dos categorías analíticas que son fundamentales para la constitución de un polo epistemológico en la Salud Colectiva: sociedad y estructura.

No existe una definición única de sociedad. Los usos más comunes son dos: 1) referido, en un sentido amplio, a la totalidad de las relaciones sociales entre las personas; 2) como "conjunto relativamente complejo de individuos de ambos los sexos y de todas las edades, permanentemente asociados y equipados de patrones culturales comunes, propios para garantir la continuidad del todo y la realización de sus ideales. En este sentido, lo más general, la sociedad alcanza diferentes grupos parciales (familia, sindicato, iglesia, etc.) que se forman dentro de ella" (19 p.139); de esa forma, la sociedad incorpora las instituciones y la cultura de un grupo.

Se sabe que en la teoría social y política de la Edad Media no había diferencia entre sociedad, como tal, y comunidad política. La distinción entre Estado y sociedad surgió gradualmente durante las convulsiones religiosas y políticas de los siglos XVI y XVII. Fue en el siglo XIX que Comte y Spencer insistieron en que la sociedad no es solo un nombre colectivo para muchos individuos, sino una "entidad" característica que transciende los individuos que a ella pertenecen. En los años '30 del siglo XX, muchos sociólogos como Ginsberg, Maclver y Parsons, sugirieron que sociedad se refería a la "tesitura total" o al "complejo esquema total" de relaciones sociales (20).

Con relación a la perspectiva adoptada por Marx, se debe recordar que él utilizó la palabra "sociedad" en tres sentidos para referirse a fenómenos distintos pero correlacionados: 1) la sociedad humana; 2) tipos históricamente existentes de sociedad (por ejemplo: la sociedad feudal); y 3) cualquier sociedad particular (por ejemplo: la Roma antigua). Como señala Bottomore:

Lo que hay de característico en la concepción de Marx es, primero, que parte de la idea de seres humanos que viven en sociedad y no involucra una antítesis entre individuo y sociedad que solo puede ser superada por la superposición de algún tipo de contrato social, o alternativamente, considerando a la sociedad como un fenómeno supraindividual. (21 p.342-343) 
Importante, también, es la idea de que tal concepción no separa la sociedad de la naturaleza y que ese intercambio se desarrolla históricamente a través del trabajo humano.

Dentro de la perspectiva que asumimos en este trabajo, escogemos como término correspondiente a sociedad, el de representación.

No se puede negar la importancia de este concepto básico, como sostienen autores como Moscovici, Jodelet, Spink, Herzlich, Minayo y otros, ni su aplicación en estudios de psicología social, antropología, sociología y ciencias políticas. Como recuerda Spinky (22), las representaciones sociales figuran entre las más tradicionales de las teorías del conocimiento. Tomando como referencia las ideas desarrolladas en Francia por Jodelet, en 1985, dice que las representaciones son

...modalidades del conocimiento práctico orientadas a la comunicación y la comprensión de la situación social, material e ideativa en la que vivimos. Son, en consecuencia, formas de conocimiento que se manifiestan como elementos cognitivos -imágenes, conceptos, categorías, teorías- pero que no se reducen jamás a los componentes cognitivos. (22 p.300)

Refuerza también un punto que es crucial en la comprensión de las representaciones:

...al ser socialmente elaboradas y compartidas, contribuyen a la construcción de una realidad común, que posibilita la comunicación. De este modo, las representaciones son esencialmente fenómenos sociales, que incluso accediendo a partir de su contenido cognitivo, deben ser entendidos a partir de su contexto de producción. O sea, a partir de las funciones simbólicas e ideológicas a las que sirven y de las formas de comunicación en que circulan. (22 p.300)

Laplantine establece una distinción que es relevante. Para él, la representación

...es el encuentro de una experiencia individual y de modelos sociales en un modo de aprehensión particular de lo real: el de la imagen-creencia, que, contrariamente al concepto y a la teoría que es su racionalización secundaria, siempre hay una tonalidad afectiva y una carga irracional. (23 p.242)
Sin duda, los conceptos con los cuales estamos lidiando se revisten de una mayor complejidad, por lo que necesitarían desarrollarse más. Sin embargo, aquí están planteados como una propuesta que nos parece interesante para la Salud Colectiva.

Con relación al tercer concepto, el de estructura, también presenta una multiplicidad de aspectos, de los que tomamos algunas ideas centrales. Pero, en primer lugar, es importante situar la posición de este concepto paradigmático, cuya historia tiene como referencia la famosa conferencia celebrada en París en 1959. En esa oportunidad, un grupo de expertos formado por Roger Bastide, Claude Lévi-Strauss, François Perroux, Arthur Henry Lefebvre, Raymond Aron, Pierre Vilar, M. Carlos Moraza, Lucien Goldman, entre otros, se reunieron para la elaboración de un diccionario terminológico de las Ciencias Sociales. Con relación al término "estructura", el objetivo fue estudiar en detalle los posibles usos del término en diferentes áreas de la ciencia, lo que resultó en el libro coordinado por Roger Bastide (24). En sus orígenes latinos, estructura viene del verbo struere (construir) y desde el siglo $\mathrm{XVI}$, el sentido arquitectónico, dio paso a otros usos: con relación al hombre, la disposición de órganos del cuerpo humano; con relación a las obras humanas, especialmente la lengua, Bastide muestra cómo el término sedujo a anatomistas, gramáticos y luego a los estudiosos de las ciencias exactas, de las ciencias de la vida y de las ciencias del hombre y la naturaleza, marcando "una serie de posibles rutas, así como de etapas". Visto como un concepto fecundo para las disciplinas que la utilizan, en palabras de Mendonça:

El concepto de estructura incluye diferentes aplicaciones de acuerdo con el área de estudio en el que sea empleado; en lógica, en matemáticas, en psicología, en economía, en historia o en antropología no abarca una significación única. (5 p.51)

Son diversas las concepciones que se construyeron, relacionadas a la estructura. Boudon y Bourricaud sintetizan algunas de las concepciones, poniendo en evidencia la imposibilidad de hacer una lista exhaustiva de significados dado que en sociología existen sentidos tan diversos. Así, puede designar los elementos estables de un 
sistema en oposición a sus elementos variables (coyuntura). En este sentido, es interesante señalar que "la noción de estructura de un modelo, por ejemplo, designa los parámetros del modelo, o el conjunto de los parámetros y funciones" (25 p.221). En otras situaciones, como recuerdan los autores citados, es utilizada para distinguir lo fundamental de lo secundario, lo esencial de lo noesencial, lo primitivo de lo derivado. Recuerdan también, que la perspectiva marxista "explica el hecho de que los sociólogos frecuentemente emplean la noción de "estructura social" como una especie de duplicado de "sistema de estratificación", siendo las variables de estratificación consideradas entonces como primeras y determinantes" (25 p.224). Si bien hay otros significados registrados, dos nos parecen importantes: el primero se refiere a las "estructuras sociales" como sistemas de restricciones que señalan la acción individual, no dejando margen de autonomía para los individuos; el segundo, es que la noción de estructura puede aparecer en correlación con la de sistema si entendemos como tal a un conjunto de "elementos interdependientes".

Como muestra Domingues: "otro eje fundamental de análisis para la teoría sociológica es el que se acordó en denominar como relación entre estructura y acción" (7 p.17). El autor retoma la discusión de las relaciones individuo/sociedad y, entre las preguntas formuladas, resalta: "¿Cómo definir la 'acción' social?, ¿cuáles son sus aspectos fundamentales?"

A los fines de este trabajo, aunque no desarrollaremos un análisis detallado de esta cuestión, señalaremos que acción es un concepto central para la sociología, que viene de la construcción weberiana. La propia definición de la sociología, según Weber, es "...una ciencia que pretende entender la acción social, interpretándola, para de esa forma explicarla causalmente en su desarrollo y efectos". Incluso para este sociólogo, la acción social es "una acción donde el sentido conscientizado por el sujeto o los sujetos está referido a la conducta de otros, siendo orientada por ésta en sus desdoblamientos" (26 p.5).

Para Weber, la acción social no es toda acción exterior, sino solo aquella que, aunque ocurra en la intimidad de los seres humanos, se encuentra orientada por otros actores, por lo que es clasificada en cuatro tipos: a) la acción racional con relación a los fines que se propone, determinada por expectativas o anticipaciones de comportamiento, tanto de objetos del mundo exterior como de otros hombres, y utilizando esas expectativas como condiciones o medios para lograr los fines propuestos: la planificación o cualquier proyecto, individual o social;

b) la acción racional con relación a valores, determinada por la carencia constante en el valor, ético, estético o religioso, de cierta conducta, independiente de los valores que pueda alcanzar;

c) la acción afectiva, especialmente emotiva, determinada por estados emocionales actuales;

d) la acción tradicional, determinada por una costumbre arraigada.

\section{CONCLUSIONES}

Al escoger estos tres pares de conceptos nucleares no partimos de lo que denominaríamos un "arbitrario epistemológico", pues en realidad asumimos la necesidad de tener un punto de partida, con una mínima construcción lógica que posibilite otros desdoblamientos. Asumimos, también, que los tres conceptos se interrelacionan (Figura 1).

Creemos que de esta base conceptual se desprenden otros conceptos como sujeto/actores, representación/experiencia, acción/praxis. Nos gustaría aclarar que el hecho de presentar la base conceptual bajo la forma de "paired concepts", como fueran denominados por Reinhard Bendix y Bennet Berger y citadas por Philippe Corcuff (8 p.11), no significa que estamos viendo el mundo social de manera dicotómica. Los más recientes estudios evidencian que el esfuerzo teórico ha sido el de superar las oposiciones (objetivo/subjetivo, colectivo/individual, por ejemplo) y "pensar conjuntamente aspectos de la realidad clásicamente aprendidos como antagonistas". En esta dirección, ha sido importante la perspectiva constructivista, según la cual, "las realidades sociales son aprehendidas como construcciones históricas y cotidianas de los actores individuales y colectivos" (8 p.11). 
Figura 1. INTERRELACIÓN DE LOS CONCEPTOS.

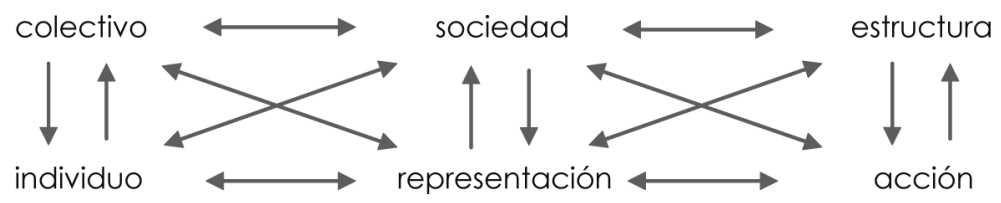

Fuente: Elaboración propia

Otra idea que nos parece extremadamente importante en la construcción de la salud, ya apuntada por nosotros, pero que merece ser enfatizada, es la relacionada al sujeto. En su trabajo, Paim y Almeida Filho señalan que hay una necesidad de
Retomar la problemática del sujeto no significa la negación de las estructuras, del mismo modo que la definición de un marco conceptual para la Salud Colectiva no implica la adopción de un cuadro teórico de referencia exclusivo y excluyente. (27 p.310)

...identificación de una "nueva positividad" en

la articulación de las dimensiones objetiva y

subjetiva en el campo social de la salud.

\section{NOTAS FINALES}

a. Este trabajo es parte del proyecto Historia de la Salud Colectiva financiado por el Conselho Nacional de Desenvolvimento Científico e Tecnológico (CNPq), beca de productividad.

\section{REFERENCIAS BIBLIOGRÁFICAS}

1. Japiassu H. Introdução ao pensamento epistemológico. Río de Janeiro: F. Alves; 1975.

2. Testa M. Pensar em saúde. Porto Alegre/Río de Janeiro: Artes Médicas, Abrasco; 1992.

3. Samaja J. Fundamentos epistemológicos de las ciencias de la salud. [Tesis de Doctorado]. Río de Janeiro: Escola Nacional de Saúde Pública; 1997.

4. Breilh J. Epidemiología nueva: construcción intercultural de otro paradigma de la ciencia.
[Tesis de Doctorado]. Salvador: Instituto de Saúde Coletiva; 2002.

5. Mendonça ND. O uso dos conceptos: uma questão de interdisciplinaridade. Bagé: FAT/UnBa; 1983.

6. Abbagnano N. Dicionário de Filosofia. San Pablo: Martins Fontes; 1998.

7. Domingues JM. Teorias sociológicas no século XX. Río de Janeiro: Civilização Brasileira; 2001.

8. Corcuff P. As novas sociologias: construções da realidade social. Bauru: EDUSC; 2001. 
9. Durkheim E. As regras do método sociológico. En: Durkheim. San Pablo: Abril; 1978. (Coleção Os Pensadores).

10. Donnangelo MCF. A pesquisa na área da Saúde Coletiva no Brasil: a década de 70. En: Ensino da Saúde Pública, Medicina Preventiva e Social no Brasil. Río de Janeiro: Abrasco; 1983.

11. Pereira JCM. A explicação sociológica na Medicina Social. [Tesis de Libre-Docencia]. Ribeirão Preto: Faculdade de Medicina; 1983.

12. Elias N. A sociedade dos homens. Río de Janeiro: Jorge Zahar Editor; 1994.

13. Bourdieu P. Espaço social e poder simbólico. En: Bourdieu P. Coisas ditas. San Pablo: Brasiliense; 1990.

14. Giddens A. A constituiçao da sociedade. San Pablo: Martíns Fontes; 1989.

15. Berger $P$, Luckmann T. A construção social da realidade. San Pablo: Vozes; 1985.

16. Cicourel AV. Cognitive sociology: language and meaning in social interaction. Nueva York: Free Press; 1974.

17. Ciência \& Saúde Coletiva. Río de Janeiro: Abrasco. Vol. 1 No. 1, 2001.

18. Minayo MCS. Estrutura e sujeito, determinismo e protagonismo histórico: uma reflexão sobre a práxis da saúde coletiva. Ciência e Saúde Coletiva. 2001;6(1):7-19.
19. Willems E. Dicionário de Sociologia. Río de Janeiro: Editora Globo; 1950.

20. Dicionário de Ciências Sociais. Río de Janeiro: Editora da Fundação Getúlio Vargas; 1986.

21. Bottomore $T$, editor. Dicionário do pensamento marxista. Río de Janeiro: Jorge Zahar Editor. 1988.

22. Spinky MJP. O conceito de representação social na abordagem psicossoscial. Cadernos de Saúde Pública.1993;9(3):300-308.

23. Laptantine F. Antropologia dos sistemas de representações das doenças: sobre algumas pesquisas desenvolvidas na França contemporânea e examinadas à luz de uma experiência brasileira. En: Jodelet $D$, organizadora. As representações sociais. Río de Janeiro: EdUERJ; 2001.

24. Bastide R, organizador. Usos e sentido do termo estrutura. San Pablo: Editora da Universidade de São Paulo; 1971.

25. Boudon R, Bounicaud F. Dicionário Critico de Sociologia. San Pablo: Atica; 1993.

26. Weber M. Economía y sociedad. México: Fondo de Cultura Económica; 1979. vol. 1.

27. Paim JS, Almeida Filho N. Saúde coletiva: uma "nova saúde pública" ou campo aberto a novos paradigmas? Revista de Saúde Pública. 1998;32(4):299-316.

\section{FORMA DE CITAR}

Duarte Nunes E. Por una epistemología de la Salud Colectiva: los conceptos sociológicos. Salud Colectiva. 2010;6(1):11-19

Recibido el 18 de marzo de 2009

Versión final presentada el 4 de junio de 2009

Aprobado el 1 de julio de 2009 